\title{
CAPACIDADE DE INOVAÇÃO EM PMES DO SEGMENTO INDUSTRIAL DE CONFECÇÕES
}

\section{Cristina Hillen}

Mestre em Administração pela Universidade Estadual de Maringá - UEM

Professor do Ensino Superior da Universidade Estadual do Paraná - UNESPAR

cristina.hillen@gmail.com (Brasil)

\section{Hilka Pelizza Vier Machado}

Doutora em Engenharia de Produção pela Universidade Federal de Santa Catarina - UFSC

Professora do Centro Universitário Cesumar - UniCesumar

hilkavier@yahoo.com (Brasil)

\section{RESUMO}

A inovação é importante atualmente não apenas para grandes, mas também para pequenas e médias empresas (PMEs). Esta pesquisa analisou a capacidade de inovação de PMEs de indústrias de confecções do município de Maringá, considerado um pólo do vestuário paranaense e brasileiro, cujas empresas são predominantemente de pequeno e médio porte. A investigação utilizou o modelo de Julien et al. (2009). A natureza da pesquisa é exploratória e quantitativa, tendo sido realizada por meio de survey, com questionário estruturado aplicado junto a 34 empreendedores, cujos resultados mostraram que o setor apresenta baixa capacidade de inovação. As inovações apresentaram-se como fracas, não associadas à capacidade de absorção da informação.

Palavras-chaves: Inovação; Capacidade de Inovação; PMEs. 


\section{INTRODUÇÃO}

A capacidade de inovação é dependente de capital estrutural e relacional. Ela depende dos recursos financeiros, humanos, tecnológicos, informacionais, da capacidade de absorção da informação e de uma cultura organizacional que promova e gerencie competências estratégicas e organizacionais, para pôr em prática ideias e métodos diferentes, que resultem em novos produtos e processos inovadores (Damanpour \& Shneider, 2006), bem como da criação de valor (Varis \& Littunen, 2010).

A mensuração da inovação em pequenas e médias empresas (PMEs), de modo geral, considera as orientações da Organização para Cooperação Econômica e Desenvolvimento (OCDE) (2005), nas quais se incluem informações de P\&D e informações contábeis. Modelos específicos para avaliação da inovação em PMEs têm demonstrado melhor efetividade, como por exemplo, o radar de inovação, utilizado para mensurar a inovação setorial e utilizado por Oliveira, Gois, Cavalcanti, Paiva Junior e Marques (2014). Outro modelo é o de Julien, Leyronas and Moreau (2009), que busca mensurar a capacidade de absorção da informação em PMEs, tendo por base as fontes de informações organizadas em redes, a capacidade de absorção da informação e a transformação de informação em inovação. O modelo propicia a mensuração da inovação em PMEs por meio de outras bases, que não as informações de Pesquisa \& Desenvolvimento (P\&D) e contábeis, mas utiliza-se de variáveis que interferem diretamente na capacidade de inovação das empresas e que são de conhecimento dos empreendedores.

O segmento industrial de confecções de artigos do vestuário, foco do presente estudo, faz parte da cadeia produtiva da indústria têxtil e de confecção. Caracteriza-se como segmento que possui processos pouco automatizados e dependentes de mão de-obra direta, produz uma diversidade de produtos e apresenta-se como altamente competitivo. Neste segmento, de modo geral, grandes empresas dedicam-se a produtos padronizados que requerem modificações secundárias diante de mudanças da moda e de estações do ano, enquanto as PMEs voltam-se para produtos mais influenciados pelas variações da moda e pela sazonalidade (Universidade Estadual de Campinas, UNICAMP, 2008).

No Brasil, 99\% das indústrias do segmento industrial de confecções de artigos do vestuário são PMEs (Instituto Brasileiro de Geografia e Estatística, IBGE, 2012). Especificamente na região de Maringá, Estado do Paraná, considerada pólo do vestuário paranaense, essas empresas são em maioria PMEs, possuindo elevada representatividade econômica e sendo responsável por alta empregabilidade (Federação das Indústrias do Estado do Paraná, FIEP, 2011). Tais características, aliadas à inexistência 
e pesquisa sobre capacidade de inovação em PMEs, especificamente nesse segmento e nessa região deram origem a esta pesquisa, influenciada também pela representatividade regional do setor e pela importância da capacidade de inovação para essas empresas. O modelo de Julien et al. (2009) foi anteriormente utilizado no setor de vestuário junto a PMEs no Congo-Brazzaville, propiciando também uma possibilidade de comparação dos resultados.

Esta pesquisa teve por objetivo analisar a capacidade de inovação de um grupo de PMEs do segmento industrial de confecções de artigos do vestuário na região de Maringá, no Estado do Paraná, utilizando o modelo de pesquisa de capacidade de absorção da informação adaptado a PMEs, criado por Julien et al. (2009) e validado em estudos anteriores (Julien et al., 2009; Leyronas \& Moreau, 2006).

Este artigo apresenta os pressupostos que deram origem ao modelo de Julien et al. (2009), bem como o detalhamento dos procedimentos da pesquisa realizada e os resultados encontrados.

\section{CAPACIDADE DE INOVAÇÃO DE PMES}

A capacidade de inovação pode ser entendida como o conjunto de competências, conhecimentos, ferramentas e recursos financeiros. É formada pela tecnologia utilizada pela empresa, pelas habilidades inovadoras dos trabalhadores e pelos processos que diretamente promovem a inovação (Jardon, 2012; Rejeb, Guimarães, Boly \& Assiélou, 2008). Portanto, o que leva uma empresa a inovar é sua capacidade de inovar. Todas as empresas têm capacidade para inovar, sendo que os empreendedores influenciam as capacidades organizacionais, estabelecendo a cultura organizacional, motivando funcionários e capacitando-os para a mudança e para a inovação (Rejeb et al., 2008).

A continuidade e crescimento da empresa dependerão da sua capacidade de inovação em uma base contínua. O pré-requisito de cada inovação é a geração de novos conhecimentos ou a combinação de conhecimentos existentes. Cada entidade (indivíduo, grupo e organização) tem a sua própria base de conhecimento e seu próprio processo de aprendizagem, sendo que as multiplicidades de processos de aprendizagem que as organizações possuem baseiam-se em diferentes fontes de informação. E nesse sentido, cabe destacar que nem toda informação é suficiente por si mesma e supõe atualização constante da capacidade de absorção da informação por parte das organizações, o que resulta da interação de seus processos de aprendizado, da intensidade de retroações positivas e da qualidade das redes que as organizações constroem (Julien, 2010; Varis \& Littunen, 2010).

\subsection{Fontes de informações e redes}

Revista de Administração e Inovação, São Paulo, v. 12, n.4 p. 76-98, out./dez. 2015. 
Ao inovar, as PMEs processam informações de fora para dentro, buscando resolver os problemas existentes e se adaptarem ao ambiente em transformação. Também criam novos conhecimentos e informações, de dentro para fora, para redefinir tanto os problemas quanto as soluções e, nesse processo, recriar seu meio, tendo por base as fontes de informações. As fontes de informações, tomando por referência o processo de inovação, podem ser divididas em pessoais e impessoais, formais e informais (Julien, 2010) ou ainda tradicionais, não tradicionais e intermediárias (Julien et al., 2009; Leyronas \& Moreau, 2006).

As redes, por sua vez, se caracterizam como fontes de informação para a inovação. As redes suportam e criam espaços de interações, confrontos e enriquecimento de diferentes tipos de conhecimentos e habilidades disponíveis cuja interação com diversos atores enriquece a empresa a partir de uma base de conhecimento complementar a sua própria base (Julien, 2010). Elas permitem ampliar os recursos ou adaptar a informação às necessidades dos interlocutores, tanto para transmiti-la como para recebê-la, produzindo uma massa crítica que gera efeitos de sinergia propícios à inovação (Julien, 2010; Julien et al., 2009; Leyronas \& Moreau, 2006).

É no desenvolvimento de ligações estabelecidas pelas PMEs, na forma de redes, que se consegue acesso a recursos diversos e escassos, bem como, apoio ao processo de inovação. O acesso às informações atreladas aos relacionamentos com diferentes atores, incentiva o empreendedorismo e a mobilização permanente de recursos (Castilla, Granovetter \& Granovetter, 2000).

De acordo com a natureza das relações que se estabelecem as redes podem se constituir como redes pessoais de negócios, redes de negócios propriamente e redes informacionais (Julien, Andriambeloson \& Ramangalahy, 2004; Julien, 2010). Ao diversificar os relacionamentos tem-se acesso a diferentes informações independentes dos laços existentes. Informação e inovação têm relação com os tipos de laços estabelecidos e com sua qualidade. Os laços fortes, por se relacionarem a pessoas próximas, geram informações redundantes ao passo que laços fracos, que se relacionam com pessoas não tão próximas, dão acesso a informações diferenciais que possibilitam a geração de novas ideias (Julien, 2010).

Os laços fortes acentuam as relações, criando cada vez mais redundância e cada vez menos informações novas, pois criam zonas fechadas que reproduzem as mesmas informações (Julien, 2010; Julien, Andriambeloson \& Ramangalahy, 2004). Os laços fortes agem pela confiança que é criada pelas interações. E, informações novas fluem mais por meio de laços fracos e estes são mais propensos a desempenhar o papel de transmitir informação única e não redundante (Granovetter, 2005). 
Do ponto de vista econômico e no sentido informacional, as redes são de sinais fortes ou fracos ao invés de laços fortes e fracos. Como as redes de laços fortes trazem informações facilmente compreendidas, podem ser chamadas de sinais fortes. Ao passo que as redes de laços fracos proporcionam informações mais difíceis de serem compreendidas em função da desatenção ou falta de confiança, assim se caracterizam como de sinais fracos. As redes de sinais fracos requerem mais atenção e interpretação a partir do conhecimento acumulado, pois trazem informações estratégicas que levam a inovação e a melhoria de desempenho, enfim, a inovação é facilitada pelos sinais fracos (Julien et al., 2004; Julien, 2010, Leyronas \& Moreau, 2006).

Relacionando-se as fontes de informações com os sinais por ela gerados tem-se que as tradicionais são geralmente de sinais fortes. As não tradicionais são consideradas de sinais fracos. E as intermediárias, que são mais difíceis de especificar e baseadas no nível de facilidade de compreensão de sinais e dependentes da frequiência de utilização, podem fornecer sinais fracos ou fortes, porém geralmente são de sinais fracos (Julien et al., 2009; Leyronas \& Moreau, 2006).

Como explicitado anteriormente, a mudança ou a inovação é facilitada pelos sinais fracos, contudo adicionando ou questionando os sinais fortes, combinando-se e esmiuçando as informações recebidas geram-se novas ideias. Tal fato se caracteriza como a transformação da informação que tem origem na capacidade de absorção da informação ou na capacidade das empresas para processar e converter as informações em conhecimento e estratégia de ação (Cohen \& Levinthal,1990; Julien et al. 2004; Leyronas \& Moreau, 2006).

A capacidade de absorção da informação é crítica para a capacidade de inovação, pois está relacionada com a habilidade organizacional em reconhecer o valor das informações, assimilá-las e aplicá-las. Empresas com alta capacidade de absorção são proativas e hábeis em explorar oportunidades (Cohen \& Levinthal, 1990). Nesse contexto, os indivíduos recebem estímulo à inovação. Portanto, os sujeitos influenciam a capacidade de inovar, que por sua vez, influencia a performance da inovação. A inovação está ligada à capacidade de absorção da informação, sendo que ela tem relação com capital humano, ou seja, com conhecimento, nível educacional e experiências dos empreendedores e funcionários (Laforet, 2011).

\subsection{PMEs e capacidade de absorção da informação e inovação}

Ao observar o cenário econômico e a inovação necessariamente se observa as PMEs, pois em virtude de suas trajetórias diversificadas e por enfrentarem competição mais intensa, tendem a se arriscar mais e a lidar melhor com a incerteza e a diversidade, o que contribui para levá-las a percorrer o caminho da produção de inovações (Guimarães, 2011). As PMEs são vistas como intervenientes no

Revista de Administração e Inovação, São Paulo, v. 12, n.4 p. 76-98, out./dez. 2015. 
processo de inovação, caracterizando-se como impulsionadoras do desenvolvimento econômico e responsáveis por alta empregabilidade (Forsman \& Rantanen, 2011; Guimarães, 2011; Julien, 2010; Nieto \& Santamaría, 2010).

As PMEs, segundo Julien (2010), estão bem posicionadas para inovar, devido ao seu caráter empreendedor e conseqüentemente menos burocrático do que as grandes empresas. Elas implementam inovações no mercado com maior rapidez e facilidade do que grandes empresas. Uma das maiores vantagens das PMEs em relação às grandes empresas, no que diz respeito à inovação, é o comportamento sistêmico interdepartamental, que favorece o envolvimento sutil ou proximidade dos indivíduos, possibilitando trocas, o estabelecimento de relações diretas e informais com o mercado para captar as ideias e um sistema de comunicação rápido e ágil, baseado em informação tácita, proporcionando flexibilidade e encorajando a iniciativa e a criatividade (Julien, 2010). As PMEs têm flexibilidade para adaptação dos trabalhadores em projetos relacionados à inovação e estruturas de gestão menos complexas para implementação de novos projetos (Bhattacharya \& Bloch, 2004).

Porém, é importante ressaltar que o custo relativo da inovação é mais significativo para PMEs do que para grandes empresas devido à limitação de recursos disponíveis, tais como mão-de-obra, recursos financeiros e materiais (Laforet, 2011), além da desvantagem no que diz respeito aos recursos intangíveis, em função do menor acesso a gama de conhecimentos e competências de capital humano em relação às grandes empresas. Elas também encontram restrições no acesso aos recursos de qualidade para inovar e na capacidade de inovação (Julien, 2010; Nieto \& Santamaría, 2010). Apesar das limitações em termos de investimentos e facilidades de pesquisa e acesso a recursos de forma geral, a inovação em PMEs aparece de forma contínua, apresentando relativo impacto sobre o desempenho financeiro (Laforet, 2011).

Assim, a inovação em PMEs é dificultada pelas restrições de recursos. Os custos envolvidos na descoberta, avaliação, adaptação e transformação de informação para as necessidades específicas de cada empresa são considerados altos, limitando a qualidade das fontes de informações e a capacidade dessas empresas para extraírem do ambiente toda a informação que necessitam para o processo de inovar. Para minimizar os custos e aumentar a qualidade da informação muitas PMEs utilizam redes, sendo que os padrões de ligação em rede de inovação dependerão do tipo de oportunidades que elas buscam (Jong \& Hulsink, 2012).

Cabe destacar que as PMEs com maior capacidade de absorção da informação tendem a ser mais bem sucedidas, pois poderão desenvolver sua capacidade de inovação. Para PMEs, redes sociais e capacidade de absorção são fatores que maximizam o desempenho da inovação. Em geral, as PMEs

Revista de Administração e Inovação, São Paulo, v. 12, n.4 p. 76-98, out./dez. 2015. 
com maior capacidade de absorção irão se beneficiar com maior exposição aos diversos laços em redes sociais do que as empresas com menor capacidade de absorção (Ahlin, Drnovsek \& Hisrich, 2012).

\subsection{O modelo de Julien et al. (2009)}

Julien et al. (2009) criaram um modelo para mensurar a capacidade de absorção da informação adaptado às PMEs. Esse modelo tem por base as variáveis fontes de informação (variável independente), a capacidade de absorção (variável moderadora) e a inovação (variável dependente). O modelo consiste na avaliação da utilização das fontes de informações mais ou menos organizadas em redes ou conectadas diretamente ao contratante, na capacidade de absorção da informação e na transformação de informação em inovação. O modelo considera a capacidade de absorção chave para compreensão da relação entre fontes de informações e o grau de inovação, como pode ser visualizado na figura 1.

Figura 1 - Modelo de pesquisa da capacidade de absorção 


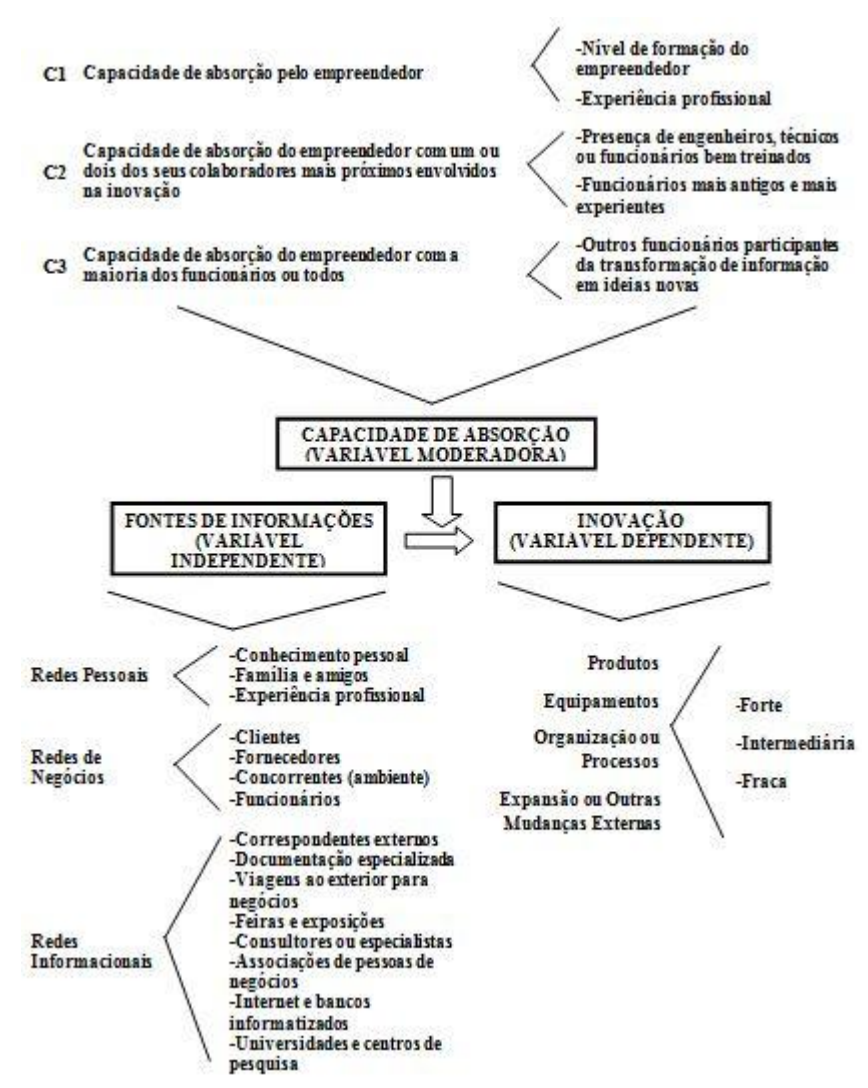

Fonte: Adaptado de Julien et al. (2009).

Com relação às fontes de informações, Julien et al. (2009) consideram três níveis: tradicionais, intermediárias e não tradicionais. Quanto às redes, consideram as redes pessoais, de negócios e informacionais, bem como, sinais fracos ou fortes que cada fonte ou rede pode gerar. Fontes tradicionais (redes pessoais) geram sinais fortes, enquanto fontes intermediárias (redes de negócios) produzem sinais fracos ou fortes e fontes não tradicionais (redes informacionais) geram sinais fracos.

Segundo Julien et al. (2009), a análise da capacidade de absorção da empresa depende da capacidade de absorção dos membros individuais (Cohen \& Levinthal, 1990), e é construída e difere entre as empresas (Lane, Balaji \& Pathak, 2006), sendo a análise dos níveis de educação do pessoal e propensão para inovar recomendados por Gray (2006). O modelo, como pode ser visualizado na figura 1, para a análise da capacidade de absorção considera três dimensões: $\mathrm{C} 1$ : a capacidade de absorção do empreendedor, apenas o tomador de decisão das PMEs; C2: a capacidade de absorção do empreendedor com um ou dois dos seus colaboradores mais próximos (profissional ou pessoal altamente experiente); e C3: a capacidade de absorção do empreendedor com a maioria ou todos os funcionários.

Por fim, com relação à inovação Julien et al. (2009) consideram mudanças nos produtos, equipamentos, organização ou processos e expansão ou outras mudanças exteriores. São considerados 
três níveis de inovações: inovação fraca; inovação intermediária e inovação forte, bem como os impactos (fortes, intermediários ou fracos) produzidos por essas inovações.

\section{PROCEDIMENTOS METODOLÓGICOS}

A pesquisa, de natureza quantitativa, consistiu em um estudo exploratório, tipo survey. Para tanto foi aplicado um questionário junto a empreendedores de PMEs do segmento industrial de confecções do vestuário na região de Maringá, Estado do Paraná, com o objetivo de analisar a capacidade de inovação dessas empresas por meio do modelo de Julien et al. (2009).

O questionário replicou o modelo criado por Julien et al. (2009), que busca identificar a capacidade de absorção como chave da relação entre informação e inovação de PMEs. Ele é composto por quatro partes, abrangendo 50 questões, distribuídas entre perguntas fechadas e escalas tipo Likert, com variação de 1 a 5 . A primeira parte do questionário incluiu perguntas sobre a estrutura da empresa e sobre o perfil do empreendedor, como: data de início das atividades, principais produtos vendidos, além de idade, nível de formação, e experiência profissional do empreendedor. A segunda parte continha questões sobre as inovações que foram feitas na empresa nos últimos dois ou três anos, sobre fontes de informações que facilitaram essas inovações, bem como condições de acesso a estas fontes e os obstáculos a essas inovações. A terceira parte incluía questões sobre as redes e as necessidades de informações habituais do negócio. A última parte do questionário abordou aspectos relativos às redes de sinal fraco ou fontes informativas "remotas" ou "não habituais".

Do universo de empresas do segmento industrial de confecções do vestuário da região de Maringá/PR, foram pesquisadas as empresas filiadas ao Sindicato da Indústria do Vestuário de Maringá (SINDVEST). O SINDVEST possuía, ano de 2012, 168 empresas filiadas, das quais 103 eram indústrias de confecções do vestuário. Destas, 34 aceitaram participar, correspondendo a uma amostra de $33 \%$. Os questionários foram aplicados junto aos empreendedores no período de outubro de 2012 a fevereiro de 2013, após ter sido traduzido e validado por meio de pré-teste realizado com 6 empreendedores.

Os dados foram organizados e tratados estatisticamente utilizando o software Excel e o SPSS 21. Como foi utilizado o modelo de pesquisa de capacidade de absorção de informação de Julien et al. (2009), a análise dos resultados foi feita em consonância com os critérios também utilizados pelos autores. Cada um dos construtos tem variáveis explicativas, distribuídas entre a capacidade de absorção (variável moderadora), as fontes de informação (variável independente); e inovação (variável dependente).

Revista de Administração e Inovação, São Paulo, v. 12, n.4 p. 76-98, out./dez. 2015. 


\section{APRESENTAÇÃO E DISCUSSÃO DOS RESULTADOS}

As empresas foram classificadas pelo número de funcionários de acordo com a OCDE (2005) para estudos sobre inovação e conforme critérios do Serviço Brasileiro de Apoio às Micro e Pequenas Empresas (SEBRAE, 2011) e Instituto Brasileiro de Geografia e Estatística (IBGE, 2012) para tamanho da empresa industrial. Assim, utilizando esses critérios as empresas, na maioria- 64,7\% são classificadas como microempresas, pois possuem menos do que 20 funcionários, conforme disposto no Quadro 1.

Quadro 1 - Classificação das empresas por número de funcionários

\begin{tabular}{|l|l|l|l|}
\hline Critérios da OCDE & Critério do SEBRAE e & \multicolumn{2}{|l|}{$\begin{array}{l}\text { Total } \\
\text { empresas }\end{array}$} \\
\hline Menos que 20 funcionários & Microempresa & 22 & $64,7 \%$ \\
\hline De 20-49 funcionários & \multirow{2}{*}{ Pequena empresa } & 3 & $8,8 \%$ \\
\cline { 3 - 5 } De 50-99 funcionários & \multirow{2}{*}{ Média empresa } & 4 & $11,8 \%$ \\
\hline De100-249 funcionários & 1 & $2,9 \%$ \\
\hline De 250-499 funcionários & & $\mathbf{3 4}$ & $\mathbf{1 0 0 \%}$ \\
\hline Total & &
\end{tabular}

Fonte: Dados da pesquisa (2013)

Quanto à idade das empresas a média encontrada foi 18 anos. Entre 3 e 10 anos estavam 29,41\% das empresas. Entre 11 e 20 anos estavam 38,24\% e 29,41\% tinha entre 21 e 30 anos. Apenas 2,94\% das empresas tinham acima de 30 anos. Sobre o percentual de empregados antigos e experientes, 73,5 \% das empresas tinha entre 1 a 10 funcionários nessa condição.

Em relação à idade dos empreendedores a média foi 42 anos. Entre 21 e 31 anos situaram-se 17,6\%; entre 32 e $42 \%$ estavam 38,2\%. Entre 43 e 53 anos foram identificados 29,4\%. Entre 54 e 64 anos o percentual foi de $11,9 \%$ e, ainda 2,9\% tinha mais do que 64 anos de idade. Quanto à experiência profissional dos empreendedores os dados mostraram que 82,4\% tinha mais do que 5 anos de experiência. Os percentuais relativos à formação educacional são apresentados no quadro 2.

Quadro 2 - Formação dos empreendedores

\begin{tabular}{|l|l|}
\hline Quesitos & $\begin{array}{l}\text { \% } \\
\text { empresas }\end{array}$ \\
\hline \% de Formação dos Empreendedores em Nível Superior & $55,9 \%$ \\
\hline \% de Formação dos Empreendedores em Pós-Graduação & $10,0 \%$ \\
\hline \% de Participação dos Empreendedores em Treinamento Adicional há menos & $21,4 \%$ \\
\hline
\end{tabular}




\begin{tabular}{|l|l|}
\hline de 1 ano & \\
\hline
\end{tabular}

Fonte: Dados da pesquisa (2013).

Observa-se que a maioria dos empreendedores possui nível superior e experiência profissional há mais de 5 anos, porém com pouca busca de educação continuada.

\section{FONTES DE INFORMAÇÕES DAS EMPRESAS}

Inicialmente foi feita análise considerando as fontes próximas ou redes pessoais, em que se destacam os conhecimentos pessoais $(82,35 \%)$ e a experiência profissional $(55,88 \%)$ como as principais fontes de que utilizam regularmente os empreendedores para procurar, transformar, desenvolver e implementar mudanças. Em relação ao nível de importância atribuído por eles para essas fontes, a família (10,98\% em média) é apontada como a de maior importância, seguida pela fonte relacionada aos conhecimentos pessoais (10,39\% em média).

Também foram analisadas as fontes intermediárias ou redes de negócios que geram sinais fracos ou fortes para promoção de mudanças na empresa. De acordo com o modelo de Julien et al. (2009) se a média da rede de negócios for maior que 1,3 ou 1,5 representa que a rede emite sinais fracos. A média obtida na pesquisa foi de 4,5 para redes de negócios, portanto para as empresas pesquisadas essas fontes emitem sinais fracos. De acordo com os resultados, para promover mudanças nos negócios, os empreendedores se utilizam regularmente de relações de negócios (67,65\%), funcionários ativos $(61,76 \%)$, clientes $(61,76 \%)$ e fornecedores $(52,94 \%)$ como principais fontes intermediárias. Quanto ao nível de importância atribuído por eles para as fontes intermediárias, concorrentes e fornecedores $(11,18 \%)$ aparecem como mais importantes, seguido de funcionários ativos $(10,78 \%)$.

Quanto às fontes distantes ou redes informacionais que geram sinais fracos, as que são as que têm maior nível de importância foram: viagens internacionais de negócios, correspondentes externos, seminários, universidades e centros de pesquisa. Com menor nível de importância foram mencionadas: documentação especializada, redes sociais informais, regulamentos e leis, pesquisa de mercado e consultores ou especialistas e são aquelas em que o empreendedor tem maior possibilidade de utilizar sua experiência profissional, seus conhecimentos pessoais e se apoiar em quadros técnicos especializados para interpretar e utilizar nos processos de mudança (Julien et al. 2004; Julien et al., 2009; Leyronas \& Moreau, 2006).

\section{Quadro 3 -Resumo das fontes de informações}

Revista de Administração e Inovação, São Paulo, v. 12, n.4 p. 76-98, out./dez. 2015. 
Capacidade de inovação em PMEs do segmento industrial de confecções

\begin{tabular}{|c|c|c|c|c|}
\hline $\begin{array}{l}\text { DIMENSÕES } \\
\text { DE ANÁLISE }\end{array}$ & \multicolumn{2}{|c|}{ INDICADORES } & $\begin{array}{c}\text { CONTAGE } \\
\text { M DE } \\
\text { RECURSOS }\end{array}$ & $\begin{array}{l}\text { MÉDIA } \\
\text { CONTAGEM } \\
\text { DE RECURSOS }\end{array}$ \\
\hline \multirow{3}{*}{$\begin{array}{c}\text { REDES } \\
\text { PESSOAIS } \\
\text { SINAIS } \\
\text { FORTES } \\
\end{array}$} & Conhecimentos pessoais & Fontes Tradicionais & 34,0 & $\overline{1,0}$ \\
\hline & Família e amigos & Fontes Tradicionais & 27,0 & 1,0 \\
\hline & Experiência profissional & Fontes Tradicionais & 34,0 & 1,0 \\
\hline \multicolumn{3}{|c|}{$\begin{array}{c}\text { TOTAL DA CONTAGEM DE RECURSOS DAS REDES } \\
\text { PESSOAIS }\end{array}$} & 95,0 & 2,8 \\
\hline \multirow{4}{*}{$\begin{array}{c}\text { REDES DE } \\
\text { NEGÓCIOS } \\
\text { SINAIS } \\
\text { FORTES OU } \\
\text { FRACOS }\end{array}$} & Clientes & Fontes Tradicionais & 44,4 & 1,3 \\
\hline & Fornecedores & Fontes Tradicionais & 42,8 & 1,3 \\
\hline & Concorrentes (ambiente) & Fontes Tradicionais & 29,0 & 0,9 \\
\hline & Funcionários & Fontes Tradicionais & 37,8 & 1,1 \\
\hline \multicolumn{3}{|c|}{$\begin{array}{l}\text { TOTAL DA CONTAGEM DE RECURSOS DAS REDES DE } \\
\text { NEGÓCIOS }\end{array}$} & 154,0 & $4,5^{*}$ \\
\hline \multirow{8}{*}{ 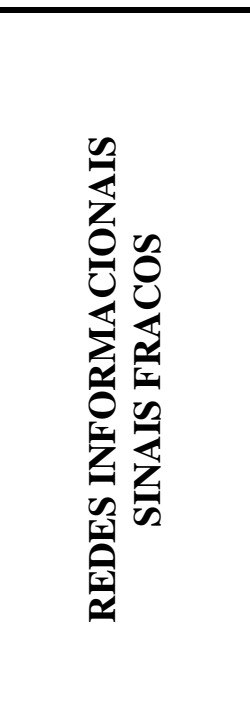 } & Correspondentes externos & $\begin{array}{l}\text { Fontes Não } \\
\text { Tradicionais }\end{array}$ & 43,0 & 1,3 \\
\hline & $\begin{array}{l}\text { Documentação } \\
\text { especializada }\end{array}$ & $\begin{array}{c}\text { Fontes } \\
\text { Intermediárias }\end{array}$ & 37,5 & 1,1 \\
\hline & $\begin{array}{l}\text { Viagens ao exterior para } \\
\text { negócios }\end{array}$ & $\begin{array}{c}\text { Fontes } \\
\text { Intermediárias }\end{array}$ & 23,0 & 0,7 \\
\hline & Feiras e exposições & $\begin{array}{c}\text { Fontes } \\
\text { Intermediárias }\end{array}$ & 109,0 & 3,2 \\
\hline & $\begin{array}{l}\text { Consultores ou } \\
\text { especialistas }\end{array}$ & $\begin{array}{l}\text { Fontes Não } \\
\text { Tradicionais }\end{array}$ & 96,0 & 2,8 \\
\hline & $\begin{array}{l}\text { Associações de pessoas de } \\
\text { negócios }\end{array}$ & $\begin{array}{c}\text { Fontes } \\
\text { Intermediárias }\end{array}$ & 42,5 & 1,3 \\
\hline & $\begin{array}{l}\text { Internet e bancos } \\
\text { informatizados }\end{array}$ & $\begin{array}{l}\text { Fontes Não } \\
\text { Tradicionais }\end{array}$ & 65,0 & 1,9 \\
\hline & $\begin{array}{c}\text { Universidades e centros de } \\
\text { pesquisa }\end{array}$ & $\begin{array}{l}\text { Fontes Não } \\
\text { Tradicionais }\end{array}$ & 16,0 & 0,5 \\
\hline \multicolumn{3}{|c|}{$\begin{array}{l}\text { TOTAL DA CONTAGEM DE RECURSOS DAS REDES } \\
\text { INFORMACIONAIS }\end{array}$} & 432,0 & 12,7 \\
\hline \multicolumn{3}{|c|}{$\begin{array}{l}\text { TOTAL DA CONTAGEM DE RECURSOS DE TODAS AS } \\
\text { REDES }\end{array}$} & 681,0 & 20,0 \\
\hline
\end{tabular}

Nota. * Média da Rede de Negócios maior que 1,3 ou 1,5 representa que a rede emite sinais fracos (JULIEN et al., 2009). Fonte: Dados da pesquisa (2013).

De forma geral, do total de recursos informacionais obtidos pelas empresas pesquisadas, $14 \%$ são das redes pessoais, $23 \%$ das redes de negócios e $63 \%$ das redes informacionais.

\section{CAPACIDADE DE ABSORÇÃO DA INFORMAÇÃO}

Sobre a capacidade de absorção da informação por parte do empreendedor (C1), identificou-se que $55,9 \%$ possuem curso superior, dos quais $10 \%$ realizaram pós-graduação; $35,3 \%$ possuem ensino 
médio, 5,9\% possuem curso técnico e 2,9\% ensino fundamental. Em termos de experiência profissional, 20,6\% dos empreendedores possuem experiência prévia no setor com menos de 5 anos e $79,4 \%$ com mais de 5 anos.

$\mathrm{Na}$ análise da capacidade de absorção da informação considerou-se, também, a presença de pessoal especializado (engenheiros, técnicos ou funcionários bem treinados e funcionários antigos e experientes - C2). Das empresas pesquisadas, 88,2\% possuem nenhum engenheiro e 11,8\% possuem apenas um. Em relação à presença de técnicos constatou-se que 67,6\% das empresas não possuem técnico, 17,6\% possuem apenas 1 técnico e 14,7\% possuem entre 2 e 4 técnicos. Em relação à presença de funcionários antigos e experientes as empresas apresentaram em média 11 funcionários. Por fim, foi constatado que na totalidade das observações a participação de outros funcionários na transformação da informação em ideias novas (C3) é nula.

Em síntese, salienta-se que 64,7\% das empresas são microempresas, com menos do que 20 funcionários e a capacidade de absorção da informação é em grande parte determinada pelo nível de qualificação dos empreendedores, pois a equipe de funcionários não contribui para a capacidade de absorção da informação e não foi observado a participação de outros funcionários.

\section{GRAU DE INOVAÇÃO}

Para análise do grau de inovação das PMEs desta pesquisa foram consideradas as inovações implementadas ao longo dos três últimos anos nas empresas. Os resultados foram: inovações em produtos $(64,7 \%)$, inovações em equipamentos $(79,5 \%)$, inovações em gestão organizacional ou processos $(85,3 \%)$. Com relação a expansão ou outras mudanças externas $(94,1 \%)$, as empresas apresentaram até 5 inovações ou mudanças.

$\mathrm{Na}$ avaliação dos empreendedores, os principais impactos das inovações implementadas nas empresas em comparação com as dos concorrentes e com as do setor foram: (a) no nível médio de rentabilidade, em 67,65\% das empresas o impacto foi médio; (b) o grau de satisfação dos clientes se apresentou forte para 55,88\% das empresas; (c) o crescimento da participação da empresa no mercado teve impacto médio em $64,71 \%$ das empresas; (d) o impacto sobre a capacidade de conquistar novos clientes se apresentou médio em $47,06 \%$ das empresas e (e) a capacidade de reter pessoal competente se apresentou como médio em 55,82\% das empresas. Assim, mesmo com baixo nível de implementação de inovações ou mudanças nas empresas (de até 5 inovações), elas apresentaram médio impacto na comparação com concorrentes e com empresas correlacionadas nos níveis de rentabilidade, satisfação de clientes, participação de mercado, conquista de novos clientes e retenção de pessoal capacitado. Do total das empresas, 32,4\% dos empreendedores desistiram de implementar inovações

Revista de Administração e Inovação, São Paulo, v. 12, n.4 p. 76-98, out./dez. 2015. 
nos três últimos anos por motivos que vão desde falta de informações e de recursos até não acreditarem no processo ou por falta de estrutura.

Quanto às inovações nos produtos, estas são consideradas médias $(55,9 \%)$ e que somente ocasionalmente elas modificam os produtos $(52,9 \%)$, expandem sua gama $(61,8 \%)$ e criam novos produtos (50\%). Em relação aos impactos das mudanças nos equipamentos, a constatação foi semelhante, ou seja, impacto médio $(52,9 \%)$ sendo que ocasionalmente ocorrem melhorias de equipamentos sugeridas pelos funcionários (70,6\%), aquisição de equipamentos usados (88,2\%), aquisição de novos equipamentos não informatizados $(70,6 \%)$ e aquisição de novos equipamentos informatizados $(79,4 \%)$. Com relação aos processos de expansão ou outra mudança externa os impactos são baixos. Quanto ao grau de informatização da contabilidade gerencial e administração esta apresentou forte impacto $-50 \%$ das empresas.

Com relação à inovação em produtos, $70,6 \%$ das empresas apresentaram inovação intermediária, enquanto $14,7 \%$ apresentaram inovação fraca e 14,7\% inovação forte. Das empresas, 97,1\% apresentaram inovação intermediária em equipamentos e 2,9\% inovação forte. Inovações em organização ou processos foram encontradas em $50 \%$ das empresas identificou-se inovações fortes, em $35,3 \%$ inovações intermediárias e em 14,7\% inovações fracas. Inovações relativas à expansão ou outra mudança externa configuraram-se em $67,6 \%$ das empresas como inovações baixas e em 32,4\% como inovações intermediárias. Em síntese, a inovação de forma geral, se apresentou como fraca, de acordo com os parâmetros do modelo de Julien et al. (2009).

\section{RELAÇÃO ENTRE FONTES DE INFORMAÇÕES, CAPACIDADE DE ABSORÇÃO DA INFORMAÇÃO E INOVAÇÃO}

A partir da mensuração das fontes de informações, da capacidade de absorção da informação e da inovação, obteve-se a contagem geral dos elementos que caracterizam as variáveis. Com base nesse total, apurou-se a média dos elementos tendo em vista uma análise geral das empresas pesquisadas (Quadro 4).

Quadro 4 - Grade Geral

\begin{tabular}{|c|c|c|c|}
\hline Recursos informacionais* & \begin{tabular}{|l|l|}
$\begin{array}{l}\text { Natureza } \\
\text { redes* }\end{array}$ & das \\
\end{tabular} & $\begin{array}{c}\text { Capacidade de } \\
\text { absorçãa* }\end{array}$ & Nível de inovação* \\
\hline Fontes de informações/média & Fontes agrupadas & $\begin{array}{l}\text { Dimensão / } \\
\text { média }\end{array}$ & Item/média \\
\hline \begin{tabular}{l|l} 
Conhecimento pessoal $^{1}$ & 1,0 \\
\end{tabular} & 2,8 & & \\
\hline
\end{tabular}

Revista de Administração e Inovação, São Paulo, v. 12, n.4 p. 76-98, out./dez. 2015. 


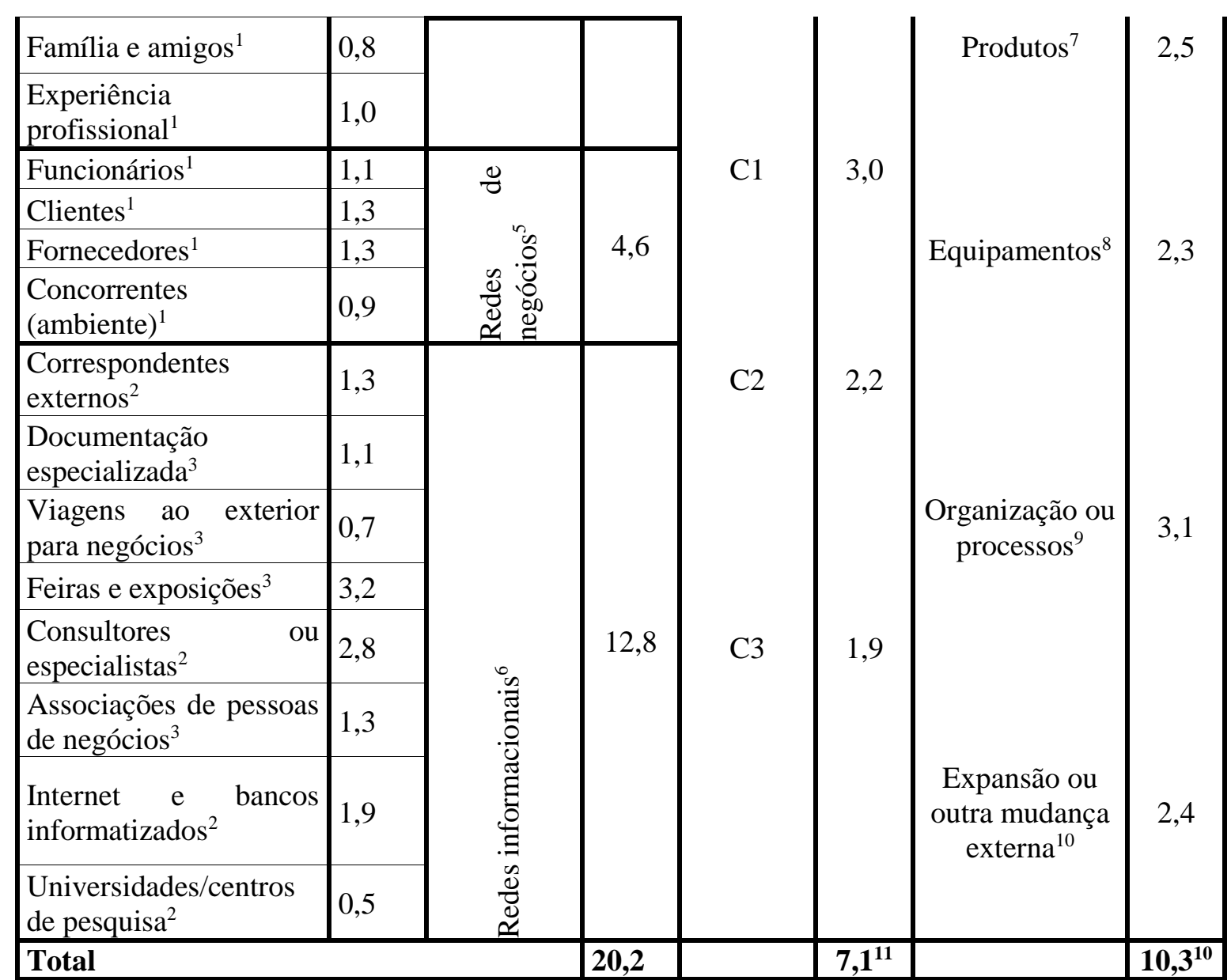

Nota. 1 Fontes Tradicionais; 2 Fontes Não Tradicionais; 3 Fontes Intermediárias; 4 Sinais Fortes; 5 Média > 1,3 ou 1,5 = Sinais Fracos; 6 Sinais Fortes; 7 Inovação Intermediária; 8 Inovação Intermediária; 9 Inovação Forte; 10 Inovação Fraca; 11 Baixa capacidade de absorção da informação.

Fonte: Dados da pesquisa (2013).

Observando os dados mostrados na grade de análise geral das variáveis (Quadro 4) conforme o modelo de Julien et al. (2009) foi realizado um estudo descritivo e inferencial dessas variáveis, a fim de investigar a correlação linear da variável independente, por meio do teste de correlação de Pearson, conforme pode ser observado nos quadros $5,6,7$ e 8 .

Quadro 5 - Correlação linear da variável independente - fontes agrupadas em redes

\begin{tabular}{|cc|c|c|c|c|}
\hline & Redes & Pessoais & Negócios & Informacionais & Informação \\
\hline \multirow{3}{*}{ Pessoais } & Correlação de Pearson & 1 & 0,175 & $-0,273$ & $-0,157$ \\
& Sig. (1 extremidade) & & 0,162 & 0,059 & 0,187 \\
& $\mathrm{~N}$ & 34 & 34 & 34 & 34 \\
\multirow{4}{*}{ Negócios } & Correlação de Pearson & 0,175 & 1 & $0,299^{*}$ & $0,484^{* *}$ \\
& Sig. (1 extremidade) & 0,162 & & 0,043 & 0,002 \\
& $\mathrm{~N}$ & 34 & 34 & 34 & 34 \\
\multirow{4}{*}{ Informacionais } & Correlação de Pearson & $-0,273$ & $\mathbf{0 , 2 9 9}$ & 1 & $0,978^{* *}$ \\
& Sig. (1 extremidade) & 0,059 & 0,043 & & 0,000 \\
& N & 34 & 34 & 34 & 34 \\
& Correlação de Pearson & $-0,157$ & $\mathbf{0 , 4 8 4 *}$ & $\mathbf{0 , 9 7 8}$ & 1 \\
& Sig. (1 extremidade) & 0,187 & 0,002 & 0,000 &
\end{tabular}

Revista de Administração e Inovação, São Paulo, v. 12, n.4 p. 76-98, out./dez. 2015. 
Capacidade de inovação em PMEs do segmento industrial de confeç̧ões

\begin{tabular}{|l|l|l|l|l|l|}
\hline $\mathrm{N}$ & 34 & 34 & 34 & 34 \\
\hline
\end{tabular}

Nota. *. A correlação é significativa no nível 0,05 (1 extremidade).

**. A correlação é significativa no nível 0,01 (1 extremidade).

Fonte: Dados da pesquisa (2013).

Ao correlacionar a variável: fontes de informações agrupadas em redes pessoais, de negócios e informacionais, não se observa correlação significativa das fontes de informações com redes pessoais. Todavia se observa uma correlação significativa entre redes de negócios e redes informacionais. As informações provenientes das redes pessoais são influenciadas pelas informações das redes de negócios e informacionais que possuem sincronismo. Tal fato favorece o processo de inovação, tendo em vista que tanto as redes de negócios quanto informacionais geram sinais fracos. Na sequiência buscou-se a correlação entre a capacidade de absorção, como mostra do quadro 6. 
Quadro 6 - Correlação linear da variável moderadora - capacidade de absorção

\begin{tabular}{|cc|c|c|c|c|}
\hline \multicolumn{2}{|c|}{ Capacidade de absorção da informação } & $\mathrm{C} 1$ & $\mathrm{C} 2$ & $\mathrm{C} 3$ & Absorção \\
\hline \multirow{4}{*}{$\mathrm{C} 1$} & Correlação de Pearson & 1 & 0,113 & 0,160 & $0,546^{* *}$ \\
& Sig. (1 extremidade) & & 0,263 & 0,183 & 0,000 \\
& $\mathrm{~N}$ & 34 & 34 & 34 & 34 \\
$\mathrm{C} 2$ & Correlação de Pearson & 0,113 & 1 & $-0,098$ & $0,542^{* *}$ \\
& Sig. (1 extremidade) & 00,263 & & 0,291 & 0,000 \\
& $\mathrm{~N}$ & 34 & 34 & 34 & 34 \\
$\mathrm{C} 3$ & Correlação de Pearson & 0,160 & $-0,098$ & 1 & $0,702^{* *}$ \\
& Sig. (1 extremidade) & 0,183 & 0,291 & & 0,000 \\
& N & 34 & 34 & 34 & 34 \\
\multirow{4}{*}{ Absorção } & Correlação de Pearson & $\mathbf{0 , 5 4 6}$ & $\mathbf{0 , 5 4 2}^{* *}$ & $\mathbf{0 , 7 0 2}^{* *}$ & 1 \\
& Sig. (1 extremidade) & 0,000 & 0,000 & 0,000 & \\
& $\mathrm{~N}$ & 34 & 34 & 34 & 34 \\
\hline
\end{tabular}

Nota. **. A correlação é significativa no nível 0,01 (1 extremidade).

Fonte: Dados da pesquisa (2013).

A correlação entre a capacidade de absorção da informação e os construtos que deram sua origem é significativa. Prevalece melhor correlação da variável capacidade de absorção da informação e capacidade de absorção do empreendedor (C1) com a maioria ou todos os funcionários (C3) e seqüencialmente com a capacidade do empreendedor $(\mathrm{C} 1)$ e do empreendedor com funcionários mais próximos envolvidos no processo de mudança (C2) - engenheiros, técnicos, funcionários bem treinados e mais antigos e experientes. Esse resultado pode ser associado ao tamanho das empresas, sendo 84\% microempresas (até 19 funcionários) e ao envolvimento dos empreendedores e da maioria dos funcionários.

Por outro lado, a correlação entre a inovação e os construtos que deram sua origem é significativa, como pode ser observado no quadro 7.

Quadro 7 - Correlação linear da variável dependente - inovação

\begin{tabular}{|c|c|c|c|c|c|c|}
\hline \multicolumn{2}{|c|}{ Inovação } & Produtos & Equipamentos & Organização & Expansão & Inovação \\
\hline \multirow{3}{*}{ Produtos } & $\begin{array}{l}\text { Correlação de } \\
\text { Pearson }\end{array}$ & 1 & 0,267 & 0,197 & 0,291 & $0,446^{* *}$ \\
\hline & $\begin{array}{c}\text { Sig. }(2 \\
\text { extremidades) }\end{array}$ & & 0,127 & 0,264 & 0,119 & 0,008 \\
\hline & $\mathrm{N}$ & 34 & 34 & 34 & 30 & 34 \\
\hline \multirow{3}{*}{ Equipamentos } & $\begin{array}{l}\text { Correlação de } \\
\text { Pearson }\end{array}$ & 0,267 & 1 & 0,084 & $0,401^{*}$ & $0,519^{* *}$ \\
\hline & $\begin{array}{c}\text { Sig. }(2 \\
\text { extremidades })\end{array}$ & 0,127 & & 0,635 & 0,028 & 0,002 \\
\hline & $\mathrm{N}$ & 34 & 34 & 34 & 30 & 34 \\
\hline \multirow{3}{*}{ Organização } & $\begin{array}{c}\text { Correlação de } \\
\text { Pearson }\end{array}$ & 0,197 & 0,084 & 1 & 0,104 & $0,646^{* *}$ \\
\hline & $\begin{array}{c}\text { Sig. }(2 \\
\text { extremidades }\end{array}$ & 0,264 & 0,635 & & 0,585 & 0,000 \\
\hline & $\mathrm{N}$ & 34 & 34 & 34 & 30 & 34 \\
\hline
\end{tabular}


Capacidade de inovação em PMEs do segmento industrial de confecções

\begin{tabular}{|c|c|c|c|c|c|c|}
\hline \multirow{3}{*}{ Expansão } & $\begin{array}{c}\text { Correlação de } \\
\text { Pearson }\end{array}$ & 0,291 & $0,401^{*}$ & 0,104 & 1 & $0,638^{* * *}$ \\
\hline & $\begin{array}{c}\text { Sig. }(2 \\
\text { extremidades })\end{array}$ & 0,119 & 0,028 & 0,585 & & 0,000 \\
\hline & $\mathrm{N}$ & 30 & 30 & 30 & 30 & 30 \\
\hline \multirow{3}{*}{ Inovação } & $\begin{array}{c}\text { Correlação de } \\
\text { Pearson }\end{array}$ & $0,446^{* *}$ & $0,519^{* * *}$ & $0,646^{* *}$ & $0, \mathbf{6 3 8}^{* *}$ & 1 \\
\hline & $\begin{array}{c}\text { Sig. (2 } \\
\text { extremidades) }\end{array}$ & 0,008 & 0,002 & 0,000 & 0,000 & \\
\hline & $\mathrm{N}$ & 34 & 34 & 34 & 30 & 34 \\
\hline
\end{tabular}

.* **. A correlação é significativa no nível 0,01 (2 extremidades). *. A correlação é significativa no nível 0,05 (2 extremidades).

Fonte: Dados da pesquisa (2013).

Finalmente, no quadro 8 apresentam-se os resultados da análise de correlação do conjunto das variáveis.

Quadro 8 - Correlação linear das variáveis fontes de informações, capacidade de absorção da informação e inovação

\begin{tabular}{|cc|c|c|c|}
\hline & & Informações & Absorção & Inovação \\
\hline \multirow{4}{*}{ Informações } & Pearson Correlation & 1 & 0,168 & 0,096 \\
& Sig. (2-tailed) & & 0,344 & 0,590 \\
& $\mathrm{~N}$ & 34 & 34 & 34 \\
Absorção & Pearson Correlation & 0,168 & 1 & $-0,011$ \\
& Sig. (2-tailed) & 0,344 & & 0,951 \\
& $\mathrm{~N}$ & 34 & 34 & 34 \\
Inovação & Pearson Correlation & 0,096 & $-0,011$ & 1 \\
& Sig. (2-tailed) & 0,590 & 0,951 & \\
& $\mathrm{~N}$ & 34 & 34 & 34 \\
\hline
\end{tabular}

Fonte: Dados da pesquisa (2013).

Como se visualiza no quadro 8 , não foram identificadas correlações estatisticamente significativas entre as variáveis: fontes de informações, capacidade de absorção da informação e inovação duas a duas. Não há uma associação linear entre as variáveis fontes de informações, capacidade de absorção da informação e inovação, o que existe é uma intensidade muito fraca de associação entre elas. Por exemplo, as variáveis fontes de informações e inovação (Quadro 8) apresentam o coeficiente de correlação $r=0,096$, em uma variação de 0 a 1, quanto mais próximo de zero o valor de "r" mais fraca é a associação.

Como não há correlação entre fontes de informações, capacidade de absorção da informação e inovação nas empresas, elas inovam, apesar da baixa capacidade de inovação, mas não influenciadas pela capacidade de absorção da informação. Os empreendedores utilizam as redes de sinais fracos (de 
negócios e informacionais) favoráveis à inovação, porém não captam ou não transformam as informações em ação, dada a baixa capacidade de absorção apresentada por essas empresas.

\section{CONSIDERAÇÕES FINAIS}

A análise da capacidade de inovação de um grupo de empresas do setor de confecções, mostrou que as fontes de informações próximas, sobre as quais se apóiam os empreendedores, são constituídas pela capacidade pessoal deles (conhecimento pessoal e experiência profissional), pelas redes de negócios, que se mostraram importantes são as redes constituídas por: funcionários, clientes, fornecedores e concorrentes, caracterizando-se como elementos ativos em trazer novas ideias e informações para o processo de inovação nessas empresas. Estas redes emitem sinais fracos, de acordo com o modelo de Julien et al. (2009), o que favorece a capacidade de inovação, pois as informações não são redundantes. Nas principais redes informacionais, que também beneficiam o processo de inovação (Julien et al., 2009; Leyronas \& Moreau, 2006), eles utilizam regularmente documentação especializada (mídias, revistas escritas ou eletrônicas), regulamentos e leis (técnica, segurança e saúde) e redes sociais informais (Facebook, Twintter, Linkedin). Destacando que das fontes informacionais a maioria dos empreendedores nunca recebeu informações dos correspondentes externos, nunca fez viagens internacionais para negócios, bem como nunca participou de seminários e nunca utilizou serviços de universidades ou centros de pesquisa.

Quanto à capacidade de absorção da informação, esta advém dos próprios empreendedores e de funcionários antigos e experientes, dado que a presença de técnicos especializados é baixa e que a participação de outros funcionários na transformação da informação em ideias novas é nula. Esse resultado reforça os achados de Gray (2006) e Ndiege, Herselman \& Flowerday (2012) sendo a capacidade de absorção da informação das PMEs influenciada pela capacidade de absorção do empreendedor, bem como, pelo tamanho e restrições de recursos, apresentando baixos níveis de capacidade de absorção da informação.

No que se refere à relação entre fontes de informações, capacidade de absorção da informação e inovação, e esta não foi significativa. A inovação é baixa, mas não é derivada das fontes de informações ou da capacidade de absorção da informação, que também se apresentou baixa pelo modelo de Julien et al. (2009). Isso pode estar associado ao tamanho das empresas, pois 64,7\% são microempresas.

A inovação em produtos e equipamentos na maioria das empresas apresenta-se como intermediária, de acordo com os parâmetros de Julien et al. (2009). Inovações em organização ou 
processos apresentaram-se em 50\% das empresas, sendo inovações fortes. Em 35,3\% observou-se inovações intermediárias e em 14,7\% inovações fracas. Inovações relativas à expansão ou outra mudança externa configuraram-se em 67,6\% das empresas como baixas e em 32,4\% como inovações intermediárias.

É importante salientar que PMEs com modesta capacidade de absorção tendem a ser mais reativas (Liao, Welsh \& Stoica, 2003; Heeley, 1997), o que pode explicar a tendência do segmento industrial de confecções no Brasil, caracterizando-se mais pela imitação do que pela inovação, adaptando os produtos lançados no mercado internacional de acordo com as tendências e variações de moda e sazonalidade (Costa \& Rocha, 2009; Garcia, Motta, Scur, Lupatini e Cruz-Moreira, 2005; UNICAMP, 2008;) e incorporando e adaptando tecnologias ao invés de implementar inovações originais (BNDES, 1996; Costa \& Rocha, 2009). Porém, mesmo sendo mais imitadoras do que inovadoras, essas empresas seguem em certa medida o gosto do consumidor, nível de concorrência e mudanças tecnológicas conforme resultados apresentados em pesquisa do Congo-Brazzaville (Julien et al., 2009).

O modelo de avaliação da capacidade de absorção da informação de PMEs de Julien et al. (2009) apresentou-se adaptado para análise das PMES do setor de confecções, mas para melhor validação sugere-se a aplicação em outro setor de atividades.

\section{REFERÊNCIAS}

Ahlin, B.; Drnovsek, M. \& Hisrich, R. D. (2012). Exploring the moderating effects of absorptive capacity on the relationship between social networks and innovation: a cross-cultural study. Recuperado de: http://sbaer.uca.edu/research/icsb/2012/Ahlin\%20297.pdf.

Bhattacharya, M. \& Bloch, H. (2004). Determinants of innovation. Small business economics, 22, 155162.

Banco Nacional do Desenvolvimento BNDES (1996). Análise conjuntural da indústria confeccionista brasileira.

Recuperado de: em:<http://www.bndes.gov.br/SiteBNDES/export/sites/default/bndes_pt/Galerias/Arquivos/ conhecimento/setorial/gs__tx09.pdf>.

Castilla, E. J.; Hwang, H.; Granovetter, E. \& Granovetter, M. (2000). Social networks in silicon valley. In A. LEE, C. et al. (Eds.). The silicon valley edge. Stanford: Stanford University Press, 218-247.

Cohen, W.M. \& Levinthal, D. A. (1990). Absorptive capacity: a new perspective on learning and innovation. Administrative science quarterly, 35(1), 128-152. 
Costa, A. C. R. da \& Rocha, É. R. P. da. (2009). Panorama da cadeia produtiva têxtil e de confecções e a questão da inovação. BNDES Setorial, Rio de Janeiro, (29), 159-202.

Damanpour, F. \& Schneider, M. (2006). Phases of the adoption of innovation in organizations: effects of environment, organization and top managers. British Jornal of Management, 17, 215-236.

Federação das Indústrias do Estado do Paraná - FIEP. (2011). Cadastro das indústrias, fornecedores e serviços. Curitiba: FIEP, 1 CD-ROM.

Forsman, H. \& Rantanen, H. (2011). Small manufacturing and service enterprises as innovators: a comparison by size. European Journal of Innovation Management, 14(1), 27-50.

Garcia, R.; Motta, F.; SCUR, G.; Lupatini, M. \& Cruz-Moreira, J. R. (2005). Esforços Inovativos de Empresas no Brasil: uma análise das indústrias têxtil-vestuário, calçados, móveis e cerâmica. São Paulo em Perspectiva, 19(2), 60-70, abr/jun.

Granovetter, M. S. (2005). The impact of social structure on economic outcomes. Journal of Economic perspectives. Winter, 19(1), 33-50.

Gray, C. (2006). Absorptive capacity, knowledge management and innovation in entrepreneurial small firms. International Jornal of Entrepreneurial Behaviour \& Research, 12(6), 345-360.

Guimarães, S. K. (2011). Empreendedorismo intensivo em conhecimento no Brasil. Caderno CRH, 24(63), 575-591.

Heeley, M.B. (1997). Appropriating rents from external knowledge: The impact of absorptive capacity on firm sales growth and research productivity, in: College, Babson (Ed.): Frontiers of Entrepreneurship Research, Babson Park, MA, Babson College, p. 390-404.

Instituto Brasileiro de Geografia e Estatística - IBGE. (2012). Cadastro central de empresas. Recuperado de: 〈www.sidra.ibge.gov.br/bda/tabela/protabl.asp?c=987\&z=p\&o=1\&i=P>.

Jardón, C. M. (2012). Determinantes de la capacidad de innovación em PYMES regionales. Universidade de Vigo. Departamento de economia aplicada. Working paper: 12/02, Sept. Recuperado de: <http://webs.uvigo.es/x06>.

Jong, J. P. J. \& Hulsink, W. (2012). Patterns of innovating networking in small firms. European Journal of Innovation Management, 15(3), 280-297.

Julien, P.A.; Andriambeloson, É \& Ramangalahy; C. (2004). Networks, weak signals and technological innovations among SMEs in the land-based transportation equipment sector. Entrepreneurship and Regional Development, 16, (4), 251-270.

Julien, P.-A.; Leyronas, C.; Makita, J. \& Moreau, É. (2009). La capacité d'absorption, l'élément clé dans la compréhension de la relation entre information et innovation Le cas des PME du CongoBrazzaville. Revue Internationale P.M.E., 22(2), 133-168.

Julien, P.-A. (2010). Empreendedorismo Regional e Economia do Conhecimento. São Paulo: Saraiva.

Laforet, S. (2011). A framework of organizational innovation and outcomes in SMEs. International Journal of entrepreneurial Behaviour \& Research, 17(4), 380-408. 
Lane, P. J.; Balaji, K \& Pathak, S. (2006). The reification of absorptive capacity : a critical view and rejuvenation of the construct . Academy of Management Review, 31 (4), 833-863.

Leyronas, C. \& Moreau, É. (2006). La capacité d'absorption: l'élément clé dans la compréhension de la relation entre l'information et l'innovation. Proceedings Annual conference of the Canadian Council for small business \& entrepreneurship. Trois-Rivières, Québec, Canadá, 23.

Liao, J.; Welsh, H. \& Stoica, M. (2003). Organizational absorptive capacity and firm responsiveness: an empirical investigation of growth-oriented firms. Entrepreneurship Theory and Practice, Autumn, 63-85.

Nieto, M. J. \& Santamaría, L. (2010). Technological collaboration: bridging the innovation gap between small and large firms. Journal of Small Business Management 48(1), 44-69.

Ndiege, J. R.; Herselman, M. E.; \& Flowerday, S. V. (2012). Absorptive capacity: relevancy for large and small enterprises. SA Journal of Information Management 14 (1), 520- 529.

Organização para Cooperação Econômica e Desenvolvimento. OCDE. (2005) Manual de Oslo. 3. ed. Tradução: FINEP - Financiadora de Estudos e Projetos. Brasil.

Oliveira, M.R.J. , Cavalcanti, A.M., Paiva Jr, F.G. \& Marques, D.B. (2014). Mensurando a inovação por meio do grau de inovação setorial e do característico setorial de inovação. Revista de Administração da Inovação, 11 (1), 115-137.

Serviço Brasileiro de Apoio às Micro e Pequenas Empresas. SEBRAE. (2011). Brasília. Recuperado de: www.sebrae.com.br em 14 nov. 2011.

Rejeb, H. B.; Guimarães, L. M.; Boly, V. \& Assiélou, N. D. G. (2008). Measuring innovation best practices: Improvement of an innovation index integrating threshold and synergy effects. Technovation, 28, 838-854.

Universidade Estadual de Campinas. UNICAMP (2008). Relatório de acompanhamento setorial: têxtil e confecção. Campinas.

Varis, M. \& Littunen, H. (2010). Types of innovation, sources of information and performance in entrepreneurial SMEs. European Journal of Innovation Management, 13(2), 128-154. 


\title{
INNOVATION CAPACITY IN SMEs SEGMENT INDUSTRIAL CONFECÇÕES
}

\begin{abstract}
Innovation is important today not only for large but also for small and medium enterprises (SMEs). This research analyzes the innovation capacity of SMEs in the clothing industries from Maringá, considered a pole clothing of Parana State and Brazilian, whose businesses are predominantly small and medium sized. The research used the model of Julien et al. (2009). The nature of research is exploratory and quantitative, have been conducted through a survey using a structured questionnaire addressed to 34 entrepreneurs, whose results showed that the sector has low capacity for innovation. The innovations presented themselves as weak, not associated with the absorption capacity of the information.
\end{abstract}

Keywords: Innovation; Innovation Capacity; SMEs.

Data do recebimento do artigo: 05/10/2014

Data do aceite de publicação: 12/09/2015

Revista de Administração e Inovação, São Paulo, v. 12, n.4 p. 76-98, out./dez. 2015. 